\title{
A Smoothening Method for the Piecewise Linear Interpolation
}

\author{
Beong In Yun \\ Department of Statistics and Computer Science, Kunsan National University, Gunsan 573-701, Republic of Korea
}

Correspondence should be addressed to Beong In Yun; paulllyun@gmail.com

Received 11 May 2015; Accepted 2 July 2015

Academic Editor: Xiao-wei Gao

Copyright (C) 2015 Beong In Yun. This is an open access article distributed under the Creative Commons Attribution License, which permits unrestricted use, distribution, and reproduction in any medium, provided the original work is properly cited.

\begin{abstract}
We propose a method to smoothen a piecewise linear interpolation at a small number of nodes on a bounded interval. The method employs a sigmoidal type weight function having a property that clusters most points on the left side of the interval toward 0 and those on the right side toward 1 . The proposed method results in a noninterpolatory approximation which is smooth over the whole interval. We provide an algorithm for implementing the presented smoothening method. To demonstrate usefulness of the presented method we introduce some numerical examples and investigate the results.
\end{abstract}

\section{Introduction}

It is well known that, by Weierstrass approximation theorem, every continuous function on a bounded interval can be approximated arbitrarily accurately by polynomials. Nevertheless, it is also true that there is no fixed array of interpolation points that achieves convergence for all continuous functions as mentioned in the literature [1]. To overcome this problem, the piecewise polynomial interpolation or rational function approximation may be considered. In particular, piecewise polynomial functions such as spline functions have been used in various approximation fields including computer graphics, data fitting, numerical integration, and differential equations $[2,3]$. However, it is not difficult to find troublesome examples for which the existing approximation methods will not suitably work when the number of interpolation nodes is not large enough.

In this paper, we propose a new noninterpolatory approximation method that is based on a smoothening process for the piecewise linear interpolation at a small number of nodes on a given interval. The main purpose of this work is summarized as follows:

(i) Rendering the piecewise linear interpolant smooth, that is, infinitely differentiable over the whole interval.

(ii) Improving the accuracy of the approximation over the interval except the interpolation nodes.

To fulfill this purpose, we construct a rational function denoted by $q_{n}(x)$ which smoothens all the vertices of the initial piecewise linear interpolant for $2^{n}$ nodes with a small integer $n$. From numerical results for some examples one can see that the presented method is available and comparable with existing outstanding methods.

Contents of this paper are as follows. In Section 2, we employ a weight function $w_{m}(x)$ in (1) of order $m \geq 1$ whose prototype is a sigmoidal transformation introduced by Prössdorf and Rathsfeld [4]. In Section 3, for a set of equally spaced nodes $\left\{x_{k}\right\}_{k=0}^{2^{n}}$ on a bounded interval $[a, b]$, we define a modified weight function $v_{j, k}(x)$ of an integer order $m_{j} \geq 1$. Then, for each integer $1 \leq j \leq n$, we construct piecewise smooth functions $L_{j, k}(x), k=1,2, \ldots, 2^{n-j}$, in the $j$ th smoothening step so that each $L_{j, k}(x)$ reflects the behavior of its precedents $L_{j-1,2 k-1}(x)$ and $L_{j-1,2 k}(x)$, depending on order $m_{j}$ of the associated weight function $v_{j, k}(x)$. It is found that the resultant approximation $q_{n}(x):=L_{n, 1}(x)$ is in $C^{\infty}[a, b]$ and noninterpolatory with the approximation property at the given nodes as shown in Theorem 1 . Section 4 includes numerical examples of some test functions whose results show the availability of the presented method.

\section{A Sigmoidal Type Weight Function}

For an interval $[\alpha, \beta]$ and a real number $m \geq 1$ we set a real valued function

$$
w_{m}(\alpha, \beta ; x)=\frac{(x-\alpha)^{m}}{(x-\alpha)^{m}+(\beta-x)^{m}}, \quad \alpha \leq x \leq \beta
$$


which we call a weight function of order $m$. The derivative of $w_{m}(\alpha, \beta ; x)$ with respect to $x$ is

$$
w_{m}^{\prime}(\alpha, \beta ; x)=\frac{m(\beta-\alpha)(x-\alpha)^{m-1}(\beta-x)^{m-1}}{\left\{(x-\alpha)^{m}+(\beta-x)^{m}\right\}^{2}}
$$

satisfying $w_{m}^{\prime}(\alpha, \beta ; x)>0$ for all $\alpha<x<\beta$. In addition,

$$
w_{m}^{\prime}\left(\alpha, \beta ; \frac{\alpha+\beta}{2}\right)=\frac{m}{\beta-\alpha} .
$$
below.

We summarize basic properties of $w_{m}(x):=w_{m}(\alpha, \beta ; x)$

(P1) Values of $w_{m}(x)$ at the points $x=\alpha,(\alpha+\beta) / 2, \beta$ are

$$
\begin{aligned}
w_{m}(\alpha) & =0, \\
w_{m}\left(\frac{\alpha+\beta}{2}\right) & =\frac{1}{2}, \\
w_{m}(\beta) & =1
\end{aligned}
$$

independently of the order $m$. In addition, $w_{m}(x)$ is strictly increasing from 0 to 1 on the interval $[\alpha, \beta]$ since $w_{m}^{\prime}(x)>0$ in (2).

(P2) For sufficiently large $m$ asymptotic behavior of $w_{m}(x)$ is

$$
w_{m}(x)= \begin{cases}O\left(\left(\frac{x-\alpha}{\beta-x}\right)^{m}\right), & \alpha<x<\frac{\alpha+\beta}{2} \\ 1-O\left(\left(\frac{\beta-x}{x-\alpha}\right)^{m}\right), & \frac{\alpha+\beta}{2}<x<\beta .\end{cases}
$$

(P3) $w_{m}(x)$ satisfies

$$
w_{m}(c-t)+w_{m}(c+t)=1, \quad 0 \leq t \leq \frac{(\beta-\alpha)}{2}
$$

for a central point $c=(\alpha+\beta) / 2$.

(P4) $w_{m}(x) \in C^{1}[\alpha, \beta] \cap C^{\infty}(\alpha, \beta)$ for any real $m \geq 1$.

By the change of variables $x=(\beta-\alpha) \xi+\alpha$ the weight function $w_{m}(x)$ becomes the so-called elementary sigmoidal transformation $\gamma_{m}(\xi)=\xi^{m} /\left(\xi^{m}+(1-\xi)^{m}\right), 0 \leq \xi \leq 1$, defined in [4]. In general, traditional sigmoidal transformations have been used for numerical evaluation of the singular integrals [5-7].

\section{Smoothening the Piecewise Linear Interpolation}

For a given interval $[a, b]$, in this paper, we consider a set of equally spaced nodes

$$
x_{k}=a+\left(\frac{b-a}{N}\right) k, \quad k=0,1,2, \ldots, N,
$$

where $N \geq 2$ is an integer not too large. We generalize the weight function $w_{m}$, defined in (1), on $[a, b]$ as follows. For an integer $j \geq 1$ and for an integer $m_{j} \geq 1$, define

$$
\begin{array}{r}
v_{j, k}(x):=w_{m_{j}}\left(a_{k}, b_{k} ; x\right)=\frac{\left(x-a_{k}\right)^{m_{j}}}{\left(x-a_{k}\right)^{m_{j}}+\left(b_{k}-x\right)^{m_{j}}}, \\
a \leq x \leq b,
\end{array}
$$

where $a_{k}$ and $b_{k}$ are some nodes such as $a \leq a_{k}<b_{k} \leq b$ defined by (7).

The function $v_{j, k}(x)$ with an integer order $m_{j}$ has inherited the properties $(\mathrm{P} 1)-(\mathrm{P} 4)$ of $w_{m_{j}}(x)$ on the restricted interval $[\alpha, \beta]=\left[a_{k}, b_{k}\right]$, including additional properties $(\mathrm{P} 3)^{\prime}$ and $(\mathrm{P} 4)^{\prime}$ on the whole interval $[a, b]$ below.

$(\mathrm{P} 3)^{\prime} v_{j, k}(x)$ satisfies

$$
v_{j, k}\left(c_{k}-t\right)+v_{j, k}\left(c_{k}+t\right)=1, \quad t \geq 0
$$

for a central point $c_{k}=\left(a_{k}+b_{k}\right) / 2$.

$(\mathrm{P} 4)^{\prime} v_{j, k}(x) \in C^{\infty}[a, b]$.

General behavior of $v_{j, k}(x), m_{j}=1,2,3,6$, with $\left[a_{k}, b_{k}\right]=$ $[0,2] \subset[a, b]=[-2,4]$, for example, is shown in Figure 1 . One can see that $v_{j, k}(x)$ becomes flatter outside the interval $\left[a_{k}, b_{k}\right]$ as the order $m_{j}$ goes higher. This can be surmised from property (P2).

From now on we set $N=2^{n}$ for an integer $n \geq 1$. Then, for a continuous function $f(x), a \leq x \leq b$, we define linear functions

$$
\begin{aligned}
l_{k}(x)=\frac{f\left(x_{k}\right)-f\left(x_{k-1}\right)}{x_{k}-x_{k-1}}\left(x-x_{k}\right)+f\left(x_{k}\right), & \\
k & =1,2, \ldots, N,
\end{aligned}
$$

each of which interpolates two points $\left(x_{k-1}, f\left(x_{k-1}\right)\right)$ and $\left(x_{k}, f\left(x_{k}\right)\right)$. Then we consider a piecewise linear interpolation,

$$
q_{0}(x):= \begin{cases}l_{1}(x), & x_{0} \leq x \leq x_{1} \\ l_{2}(x), & x_{1} \leq x \leq x_{2} \\ \vdots & \\ l_{N}(x), & x_{N-1} \leq x \leq x_{N} .\end{cases}
$$

To smoothen the piecewise linear interpolation $q_{0}(x)$ over the whole interval $[a, b]=\left[x_{0}, x_{N}\right]$ we propose an $n$-step strategy using the weight function $v_{j, k}(x)$ as follows.

Step 0. Setting initial lines:

$$
L_{0, k}(x)=l_{k}(x)
$$

for each $k=1,2, \ldots, 2^{n}=N$.

Step 1. Smoothening vertices of $q_{0}(x)$ at the nodes $x_{2 k-1}, k=$ $1,2, \ldots, 2^{n-1}$ : set

$$
\begin{array}{r}
L_{1, k}(x)=\left\{1-v_{1, k}(x)\right\} L_{0,2 k-1}(x)+v_{1, k}(x) L_{0,2 k}(x), \\
x_{2(k-1)} \leq x \leq x_{2 k}
\end{array}
$$




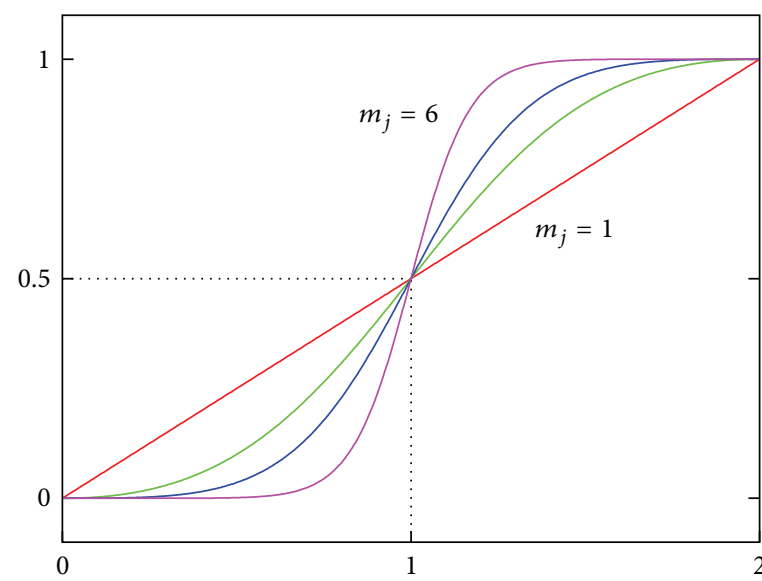

(x)

(a)

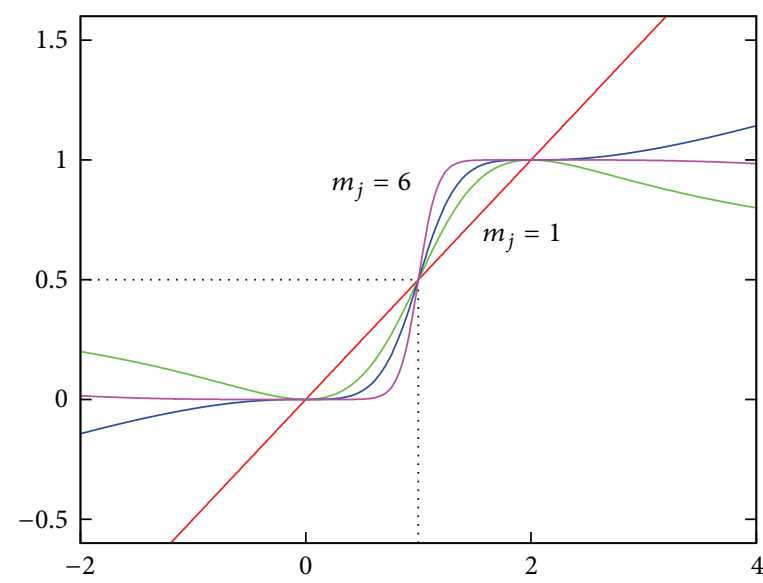

(x)

(b)

FIGURE 1: Graphs of the functions $v_{j, k}(x)$ with $a_{k}=0$ and $b_{k}=2$ for each $m_{j}=1,2,3,6$ on a subinterval $\left[a_{k}, b_{k}\right]=[0,2]$ in $(\mathrm{a})$ and those on the whole interval $[a, b]=[-2,4]$ in (b).

for each $k=1,2, \ldots, 2^{n-1}$, and define a function

$$
q_{1}(x):= \begin{cases}L_{1,1}(x), & x_{0} \leq x \leq x_{2} \\ L_{1,2}(x), & x_{2}<x \leq x_{4} \\ \vdots & \\ L_{1,2^{n-1}}(x), & x_{N-2}<x \leq x_{N} .\end{cases}
$$

Step 2. Smoothening vertices of $q_{0}(x)$ at the nodes $x_{4 k-2}, k=$ $1,2, \ldots, 2^{n-2}$ : set

$$
\begin{array}{r}
L_{2, k}(x)=\left\{1-v_{2, k}(x)\right\} L_{1,2 k-1}(x)+v_{2, k}(x) L_{1,2 k}(x), \\
x_{4(k-1)} \leq x \leq x_{4 k}
\end{array}
$$

for each $k=1,2, \ldots, 2^{n-2}$, and define a function

$$
q_{2}(x):= \begin{cases}L_{2,1}(x), & x_{0} \leq x \leq x_{4} \\ L_{2,2}(x), & x_{4}<x \leq x_{8} \\ \vdots & \\ L_{2,2^{n-2}}(x), & x_{N-4}<x \leq x_{N} .\end{cases}
$$

Step $n-1$. Smoothening vertices of $q_{0}(x)$ at the nodes $x_{2^{n-1} k-2^{n-2}}, k=1,2$ : set

$$
\begin{aligned}
L_{n-1, k}(x)= & \left\{1-v_{n-1, k}(x)\right\} L_{n-2,2 k-1}(x) \\
& +v_{n-1, k}(x) L_{n-2,2 k}(x),
\end{aligned}
$$

$x_{2^{n-1}(k-1)} \leq x \leq x_{2^{n-1} k}$, for each $k=1,2$, and define a function

$$
q_{n-1}(x):= \begin{cases}L_{n-1,1}(x), & x_{0} \leq x \leq x_{2^{n-1}} \\ L_{n-1,2}(x), & x_{2^{n-1}}<x \leq x_{N} .\end{cases}
$$

Step $n$. Smoothening a vertex of $q_{0}(x)$ at the node $x_{2^{n-1}}$ : define a final function

$$
\begin{aligned}
q_{n}(x) & :=L_{n, 1}(x) \\
& =\left\{1-v_{n, 1}(x)\right\} L_{n-1,1}(x)+v_{n, 1}(x) L_{n-1,2}(x), \\
a=x_{0} \leq x & \leq x_{N}=b .
\end{aligned}
$$

In each $j$ th smoothening step $(j=1,2, \ldots, n)$ above, function $v_{j, k}(x)$ is defined by (8) with an appropriate integer order $m_{j} \geq 1$ and nodes set by

$$
\begin{aligned}
& a_{k}=x_{2^{j}(k-1)} \\
& b_{k}=x_{2^{j} k}
\end{aligned}
$$

for each $k=1,2, \ldots, 2^{n-j}\left(=N / 2^{j}\right)$.

As a result, every vertex of the initial piecewise linear interpolation $q_{0}(x)$ has been smoothened by $q_{n}(x)$, whereas the interpolation property may be weakened as $q_{n}\left(x_{i}\right) \approx$ $f\left(x_{i}\right)$ for all $x_{i}$ 's except $x_{0}, x_{2^{n-1}}$, and $x_{N}=x_{2^{n}}$ as shown in Theorem 1. In fact, property (P4)' implies that $q_{n}(x) \in$ $C^{\infty}[a, b]$.

For intuitional comprehension on the presented method, Figure 2 is included. It shows the effect of $L_{1, k}(x)$ in the first smoothening step $(j=1)$ by comparing results for the orders $m_{1}=1,2,4,8$ of the associated weight function $v_{1, k}(x)$. One can see that $L_{1, k}(x)$ reflects the behavior of its precedents $L_{0,2 k-1}(x)$ and $L_{0,2 k}(x)$ over the larger region as order $m_{1}$ goes higher, preserving the interpolation and the smoothness on the doubled local interval $\left[x_{2(k-1)}, x_{2 k}\right]$. In general, each $j$ th piecewise smooth function $L_{j, k}(x), k=1,2, \ldots, 2^{n-j}$, has the same property on the local interval $\left[x_{2^{j}(k-1)}, x_{2^{j} k}\right]$.

Referring to the aforementioned comments, we may consider that, for small $j$ (i.e., for the small subintervals $\left.x_{2^{j}(k-1)} \leq x \leq x_{2^{j} k}\right)$, order $m_{j}$ of the weight function $v_{j, k}(x)$ might be low in order to sustain the natural behavior in 


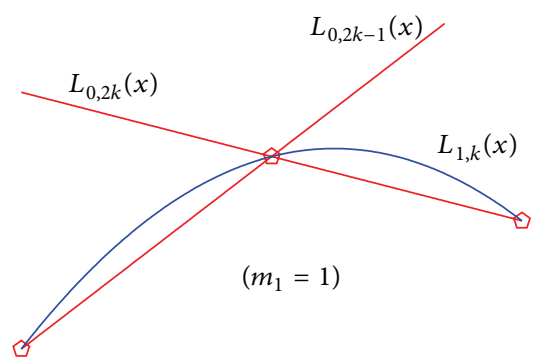

(a)

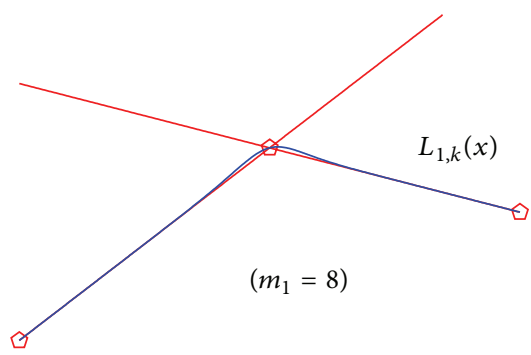

(c)

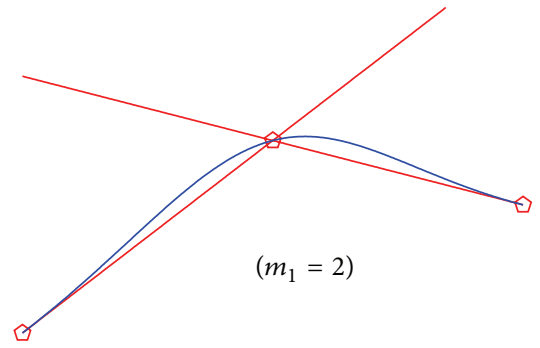

(b)

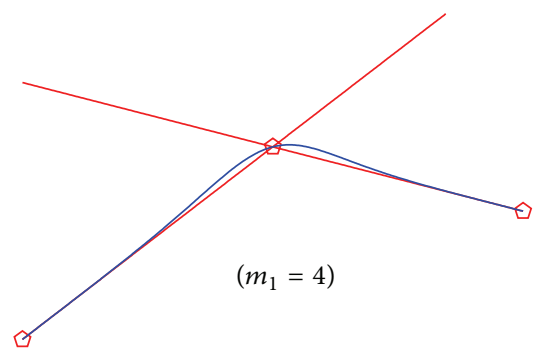

(d)

FIGURE 2: Effect of the first step $(j=1)$ smoothening $L_{1, k}(x)$ for each of the orders $m_{1}=1,2,4,8$ of the weight function $v_{1, k}(x)$.

the range between interpolation nodes. For large $j$ (i.e., for the large subintervals), higher orders are required to prevent loss of the accuracy at the nodes except the endpoints and the midpoint of the subinterval. Thus, for practical performance of the presented method, we suggest integer orders $m_{j}, j=$ $1,2, \ldots, n$, as

$$
1 \leq m_{1}<m_{2}<\cdots<m_{n} .
$$

Theorem 1. For integer orders $m_{j}$, satisfying (21), of the weight functions $v_{j, k}(x), j=1,2, \ldots, n$, with $N=2^{n}$ in (8), we have

$$
\begin{aligned}
& q_{n}\left(x_{i}\right)=f\left(x_{i}\right), \quad i=0,2^{n-1}, 2^{n}, \\
& \left|q_{n}\left(x_{i}\right)-f\left(x_{i}\right)\right| \\
& \quad= \begin{cases}O\left(h_{i}^{m_{2}}\right), & i=1,3,5, \ldots, N-1 \\
O\left(h_{i}^{m_{3}}\right), & i=2,4,6, \ldots, N-2,\end{cases}
\end{aligned}
$$

where $0<h_{i}<1$.

Proof. For each $x=x_{0}=a$ and $x=x_{2^{n}}=b$, we have

$$
\begin{aligned}
q_{n}(a) & =L_{n-1,1}(a)=L_{n-2,1}(a)=\cdots=L_{0,1}(a) \\
& =l_{1}(a)=f(a) \\
q_{n}(b) & =L_{n-1,2}(b)=L_{n-2,4}(b)=\cdots=L_{0,2^{n}}(b) \\
& =l_{2^{n}}(b)=f(b)
\end{aligned}
$$

from the definition of $L_{j, k}(x)$ and property (P1) of the weight function $v_{j, k}(x)$. Similarly, for the midpoint $x=x_{2^{n-1}}$,

$$
\begin{aligned}
q_{n}\left(x_{2^{n-1}}\right) & =L_{n-1,1}\left(x_{2^{n-1}}\right)=L_{n-2,2}\left(x_{2^{n-1}}\right)=\cdots \\
& =L_{0,2^{n-1}}\left(x_{2^{n-1}}\right)=l_{2^{n-1}}\left(x_{2^{n-1}}\right)=f\left(x_{2^{n-1}}\right) .
\end{aligned}
$$

To prove the approximation property of $q_{n}(x)$ at interior points $x_{i}, 1 \leq i \leq 2^{n}-1=N-1$, we first consider the case of $i=1$. Definition of $L_{j, k}(x)$ with the related nodes $a_{k}$ and $b_{k}$ in (20) and the assumption (21) for the order of $v_{j, k}(x)$ imply that

$$
\begin{aligned}
q_{n}\left(x_{1}\right)= & \left\{1-v_{n, 1}\left(x_{1}\right)\right\} L_{n-1,1}\left(x_{1}\right) \\
& +v_{n, 1}\left(x_{1}\right) L_{n-1,2}\left(x_{1}\right) \\
= & L_{n-1,1}\left(x_{1}\right)+O\left(v_{n, 1}\left(x_{1}\right)\right) \\
= & \left\{1-v_{n-1,1}\left(x_{1}\right)\right\} L_{n-2,1}\left(x_{1}\right) \\
& +v_{n-1,1}\left(x_{1}\right) L_{n-2,2}\left(x_{1}\right)+O\left(v_{n, 1}\left(x_{1}\right)\right) \\
= & L_{n-2,1}\left(x_{1}\right)+O\left(v_{n-1,1}\left(x_{1}\right)\right)=\cdots \\
= & L_{1,1}\left(x_{1}\right)+O\left(v_{2,1}\left(x_{1}\right)\right) \\
= & \left\{1-v_{1,1}\left(x_{1}\right)\right\} L_{0,1}\left(x_{1}\right)+v_{1,1}\left(x_{1}\right) L_{0,2}\left(x_{1}\right) \\
& +O\left(v_{2,1}\left(x_{1}\right)\right)=f\left(x_{1}\right)+O\left(v_{2,1}\left(x_{1}\right)\right),
\end{aligned}
$$

as $m_{2}$ goes to the infinity, where the last equality results from $L_{0,1}\left(x_{1}\right)=L_{0,2}\left(x_{1}\right)=f\left(x_{1}\right)$. Thus, it follows that

$$
\left|q_{n}\left(x_{1}\right)-f\left(x_{1}\right)\right|=O\left(v_{2,1}\left(x_{1}\right)\right) .
$$

In fact, since $a_{1}=x_{0}$ and $b_{1}=x_{4}$ in (20) for definition of $v_{2,1}(x)$,

$$
x_{1}<\frac{a_{1}+b_{1}}{2}=x_{2} .
$$

Then from property (P2) of the weight function $v_{2,1}(x)$ of order $m_{2}$ with $\alpha=a_{1}$ and $\beta=b_{1}$ we have

$$
v_{2,1}\left(x_{1}\right)=O\left(h_{1}^{m_{2}}\right) \text {, }
$$




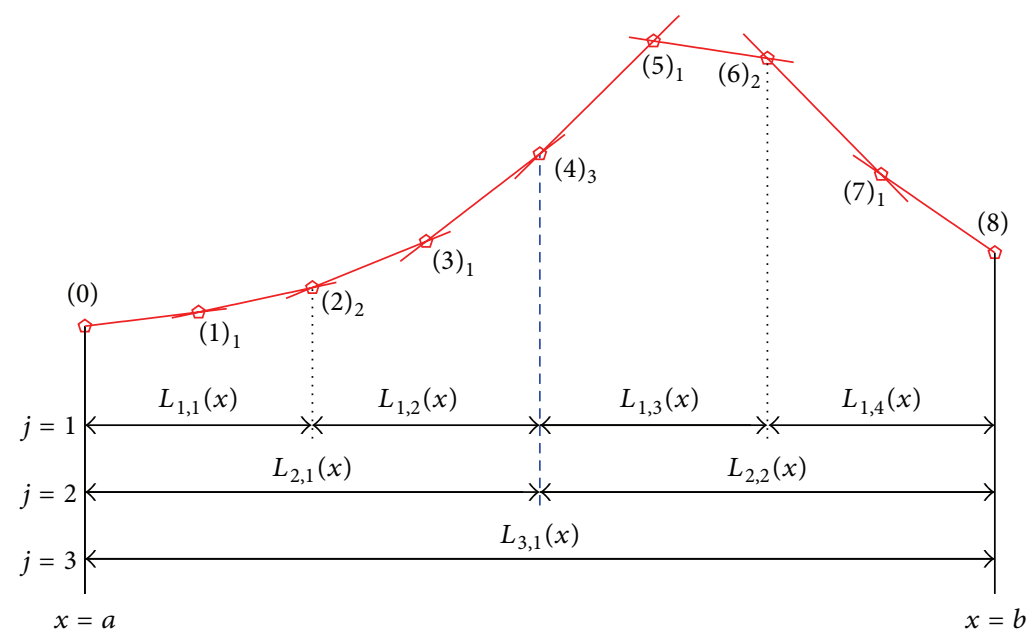

Figure 3: Illustration of the presented method for the case of $n=3$. The symbol $(t)_{j}$ denotes an interpolation point at the $t$ th node $x_{t}$ which is smoothened by $L_{j, k}(x)$ in the $j$ th smoothening step.

where $0<h_{1}<1$. This results in

$$
\left|q_{n}\left(x_{1}\right)-f\left(x_{1}\right)\right|=O\left(h_{1}^{m_{2}}\right) \text {. }
$$

By a similar way, we have, for any odd integer $i=2 k-1(k=$ $\left.1,2,3, \ldots, 2^{n-1}\right)$,

$$
\left|q_{n}\left(x_{i}\right)-f\left(x_{i}\right)\right|=O\left(h_{i}^{m_{2}}\right), \quad 0<h_{i}<1 .
$$

For the case of $i=2$, an even number,

$$
\begin{aligned}
q_{n}\left(x_{2}\right)= & \left\{1-v_{n, 1}\left(x_{2}\right)\right\} L_{n-1,1}\left(x_{2}\right) \\
& +v_{n, 1}\left(x_{2}\right) L_{n-1,2}\left(x_{2}\right) \\
= & L_{n-1,1}\left(x_{2}\right)+O\left(v_{n, 1}\left(x_{2}\right)\right) \\
= & \left\{1-v_{n-1,1}\left(x_{2}\right)\right\} L_{n-2,1}\left(x_{2}\right) \\
& +v_{n-1,1}\left(x_{2}\right) L_{n-2,2}\left(x_{2}\right)+O\left(v_{n, 1}\left(x_{2}\right)\right) \\
= & L_{n-2,1}\left(x_{2}\right)+O\left(v_{n-1,1}\left(x_{2}\right)\right)=\cdots \\
= & L_{2,1}\left(x_{2}\right)+O\left(v_{3,1}\left(x_{2}\right)\right) \\
= & \left\{1-v_{2,1}\left(x_{2}\right)\right\} L_{1,1}\left(x_{2}\right)+v_{2,1}\left(x_{2}\right) L_{1,2}\left(x_{2}\right) \\
& +O\left(v_{3,1}\left(x_{2}\right)\right)=f\left(x_{2}\right)+O\left(v_{3,1}\left(x_{2}\right)\right),
\end{aligned}
$$

where the last equality results from $L_{1,1}\left(x_{2}\right)=L_{1,2}\left(x_{2}\right)=$ $f\left(x_{2}\right)$. Thus

$$
\left|q_{n}\left(x_{2}\right)-f\left(x_{2}\right)\right|=O\left(v_{3,1}\left(x_{2}\right)\right) .
$$

Since $a_{1}=x_{0}$ and $b_{1}=x_{8}$ in (20) for definition of $v_{3,1}(x)$,

$$
x_{2}<\frac{a_{1}+b_{1}}{2}=x_{4} .
$$

Then from property (P2) of the weight function $v_{3,1}(x)$ of order $m_{3}$ with $\alpha=a_{1}$ and $\beta=b_{1}$ we have

$$
v_{3,1}\left(x_{2}\right)=O\left(h_{2}^{m_{3}}\right), \quad 0<h_{2}<1 \text {. }
$$

Thus

$$
\left|q_{n}\left(x_{2}\right)-f\left(x_{2}\right)\right|=O\left(h_{2}^{m_{3}}\right)
$$

By a similar way, we have, for any even integer $i=2 k(k=$ $\left.1,2,3, \ldots, 2^{n-1}-1\right)$,

$$
\left|q_{n}\left(x_{i}\right)-f\left(x_{i}\right)\right|=O\left(h_{i}^{m_{3}}\right), \quad 0<h_{i}<1 .
$$

The proof is completed.

The concept of the presented method for a case of $N=$ $8(n=3)$ is illustrated in Figure 3. Therein one can see that nonsmooth vertices at the nodes $x_{1}, x_{2}, \ldots, x_{7}$ appear in the initial piecewise linear interpolation. Figure 3 explains that the vertices at the nodes $x_{1}, x_{3}, x_{5}$, and $x_{7}$ are blunted in the first smoothening step $(j=1)$ and those at the nodes $x_{2}$ and $x_{6}$ are blunted in the second step $(j=2)$. Finally, the remaining vertex at $x_{4}$ is blunted in the last step $(j=n=3)$.

For implementation of the presented method, we write the following algorithm with a given data $\left\{\left(x_{i}, f\left(x_{i}\right)\right)\right.$ । $\left.i=0,1,2, \ldots, 2^{n}\right\}$ and properly chosen orders $m_{j}, j=$ $1,2,3, \ldots, n$, based on condition (21):

$$
i:=0 \text {. }
$$

While $\left(i=i+1 \leq 2^{n}\right)$.

$$
\begin{aligned}
& \text { Set } L_{0, i}(x):=\left(\left(f\left(x_{i}\right)-f\left(x_{i-1}\right)\right) /\left(x_{i}-x_{i-1}\right)\right)(x- \\
& \left.x_{i}\right)+f\left(x_{i}\right) \\
& \quad j:=0 .
\end{aligned}
$$

While $(j=j+1 \leq n)$

$$
k:=0 \text {. }
$$




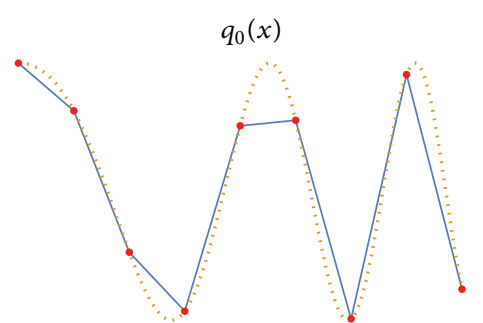

(a)

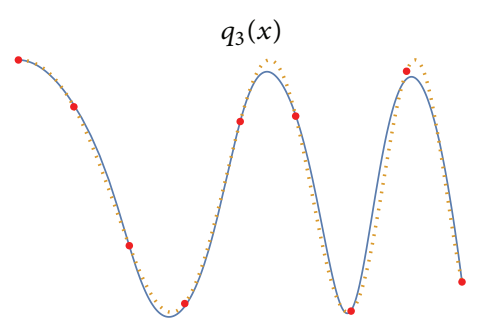

(c)

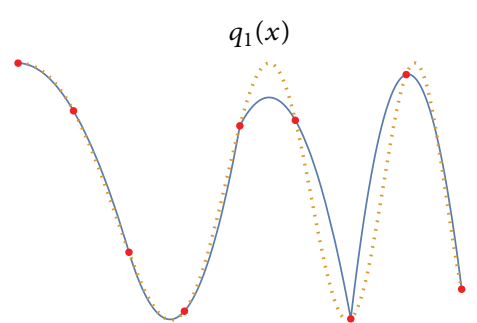

(b)

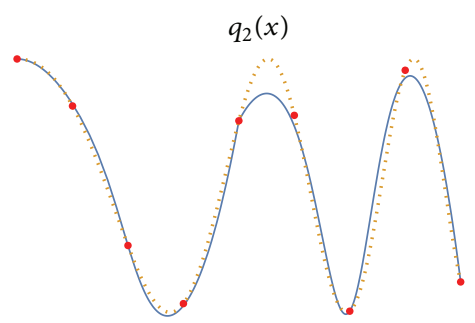

(d)

FIGURE 4: Smoothened piecewise linear interpolation $q_{j}(x), j=0,1,2,3$, for the example $f(x)$ with $N=2^{3}$. The dotted curve indicates the original graph of $f(x)$.

$$
\begin{aligned}
\text { While }( & \left.k=k+1 \leq 2^{n-j}\right) \\
a_{k}:= & x_{2^{j}(k-1)} ; \\
b_{k}:= & x_{2^{j} k} \\
v_{j, k}(x):= & \frac{\left(x-a_{k}\right)^{m_{j}}}{\left(x-a_{k}\right)^{m_{j}}+\left(b_{k}-x\right)^{m_{j}}} \\
L_{j, k}(x):= & \left\{1-v_{j, k}(x)\right\} L_{j-1,2 k-1}(x) \\
& +v_{j, k}(x) L_{j-1,2 k}(x) .
\end{aligned}
$$

Set the $j$ th smoothening interpolation,

$$
q_{j}(x):= \begin{cases}L_{j, 1}(x), & \text { if } a_{1} \leq x \leq b_{1} \\ L_{j, 2}(x), & \text { if } a_{2} \leq x \leq b_{2} \\ \vdots & \\ L_{j, 2^{n-j}}(x), & \text { if } a_{2^{n-j}} \leq x \leq b_{2^{n-j}} .\end{cases}
$$

\section{Examples}

To perform the presented algorithm we used a Mathematica (V.10) programming code, given in Appendix, where we have taken linearly increasing orders $m_{j}=3 j-2$ for the weight functions $v_{j, k}(x)$.

We choose a test function below. Consider

$$
f(x)=\cos \left(2 x+x^{2}\right), \quad 0 \leq x \leq 3
$$

with $2^{n}+1$ nodes $(n=3)$

$$
x_{k}=\frac{3}{8} k, \quad k=0,1,2, \ldots, 8 .
$$

Results of the presented method are given in Figure 4, which shows consistency with the illustration given in Figure 3 and Theorem 1. The dotted curve indicates the original graph of $f(x)$, and it is observed that $q_{j}(x), j=1,2,3$, have highly improved the accuracy of the initial piecewise linear approximation $q_{0}(x)$. In Figure 5, the resultant approximation $q_{3}(x)$ is compared with two existing approximations, the Hermite interpolation $H(x)$ and the cubic spline interpolation $S(x)$. One can see that $q_{3}(x)$ is better than $H(x)$ and $S(x)$ in approximation over the whole interval, while the interpolation property at the given nodes is weakened as shown in Theorem 1. In fact, computation of the $l_{2}$-norm error of the presented approximation $q_{3}(x)$ at the nodes $\left\{x_{k}\right\}_{k=0}^{8}$ results in $\sqrt{\sum_{k=0}^{8}\left\{f\left(x_{k}\right)-q_{3}\left(x_{k}\right)\right\}^{2}} \approx 0.068$.

For another example, we take a parametric function

$$
\begin{aligned}
r(t)=(x(t), y(t))=\left(\frac{t}{2}+\cos t, \frac{t}{2}-\sin t\right) & \\
& -\pi \leq t \leq 4 \pi
\end{aligned}
$$

with the nodes

$$
t_{k}=-\pi+\left(\frac{5 \pi}{8}\right) k, \quad k=0,1,2, \ldots, 8 .
$$

In this case, the presented method is applied to both functions $x(t)$ and $y(t)$. Figures 6 and 7 include the results of the presented method, which shows the same availability and efficiency of the method as the case of the previous test function.

\section{Conclusions}

In this paper, we proposed a method which results in the noninterpolatory approximations $q_{j}(x), j=1,2, \ldots, n$, and 


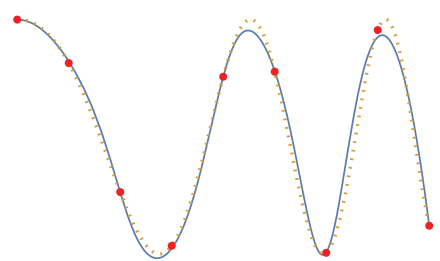

(a) $q_{3}(x)$

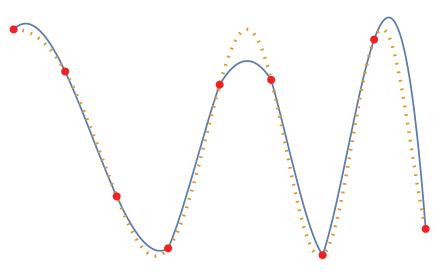

(b) $H(x)$

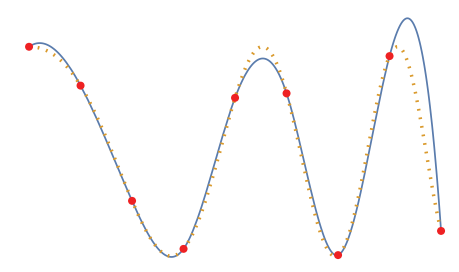

(c) $S(x)$

FIGURE 5: Comparison of the presented approximation $q_{3}(x)$, Hermite interpolation $H(x)$, and spline interpolation $S(x)$ for $f(x)$ with $N=2^{3}$. Dotted curves indicate the original graph of $f(x)$.

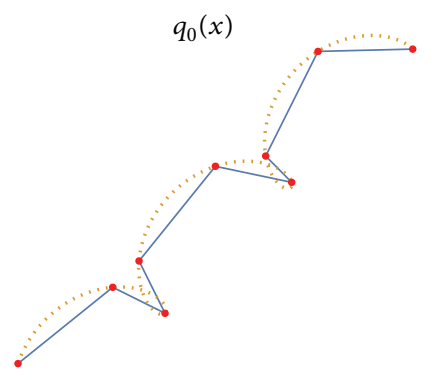

(a)

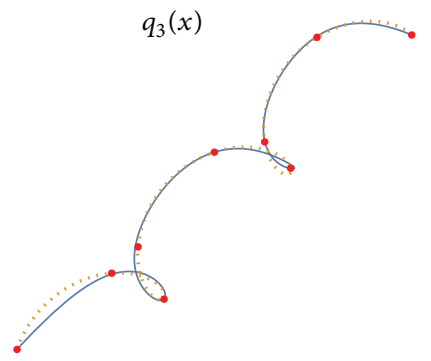

(c)

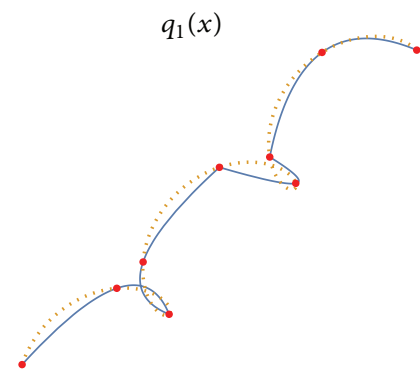

(b)

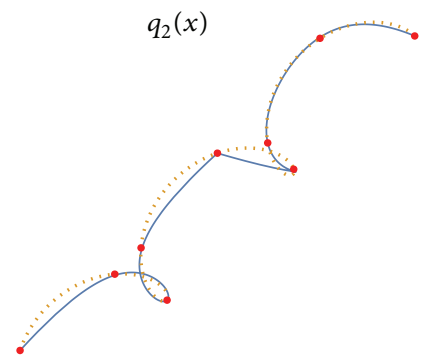

(d)

FIGURE 6: Smoothened piecewise linear interpolations $q_{j}(t), j=0,1,2,3$, for the parametric function $r(t)$ with $N=2^{3}$. The dotted curve indicates the original graph of $r(t)$.

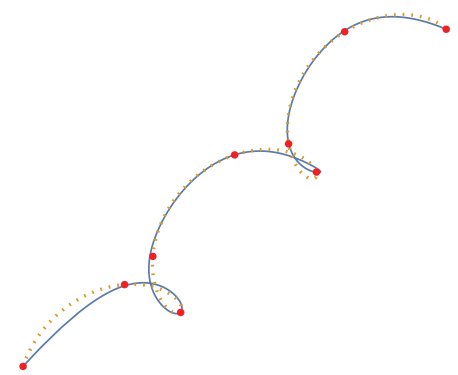

(a) $q_{3}(t)$

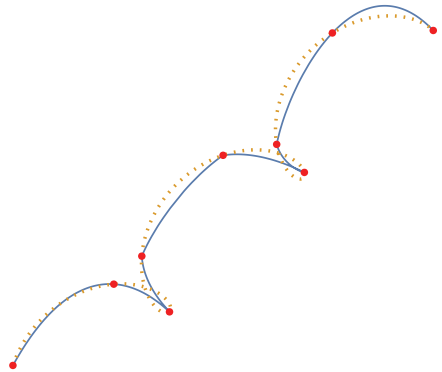

(b) $H(t)$

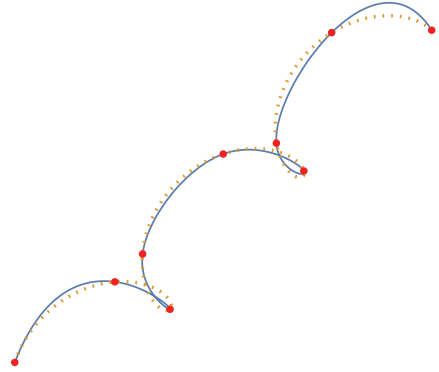

(c) $S(t)$

FIGURE 7: Comparison of the presented approximation $q_{3}(t)$, Hermite interpolation $H(t)$, and spline interpolation $S(t)$ for the parametric function $r(t)$ with $N=2^{3}$. Dotted curves indicate the original graph of $r(t)$.

the last one $q_{n}(x)$ smoothens all the vertices of the initial piecewise linear interpolant $q_{0}(x)$. Although the interpolation property of $q_{0}(x)$ at most nodes is weakened by $q_{n}(x)$ as the approximation property in Theorem 1 , we have obtained smoothness over the whole interval. Moreover, in the results of the numerical implementation for some examples, we have found that the accuracy of the presented approximation $q_{n}(x)$ is somewhat better than that of the Hermite interpolation and the spline interpolation over the whole interval.

The performance of the presented method depends on the orders of the associated weight functions, and analysis for the problem of choosing optimal orders is left as a further work. 


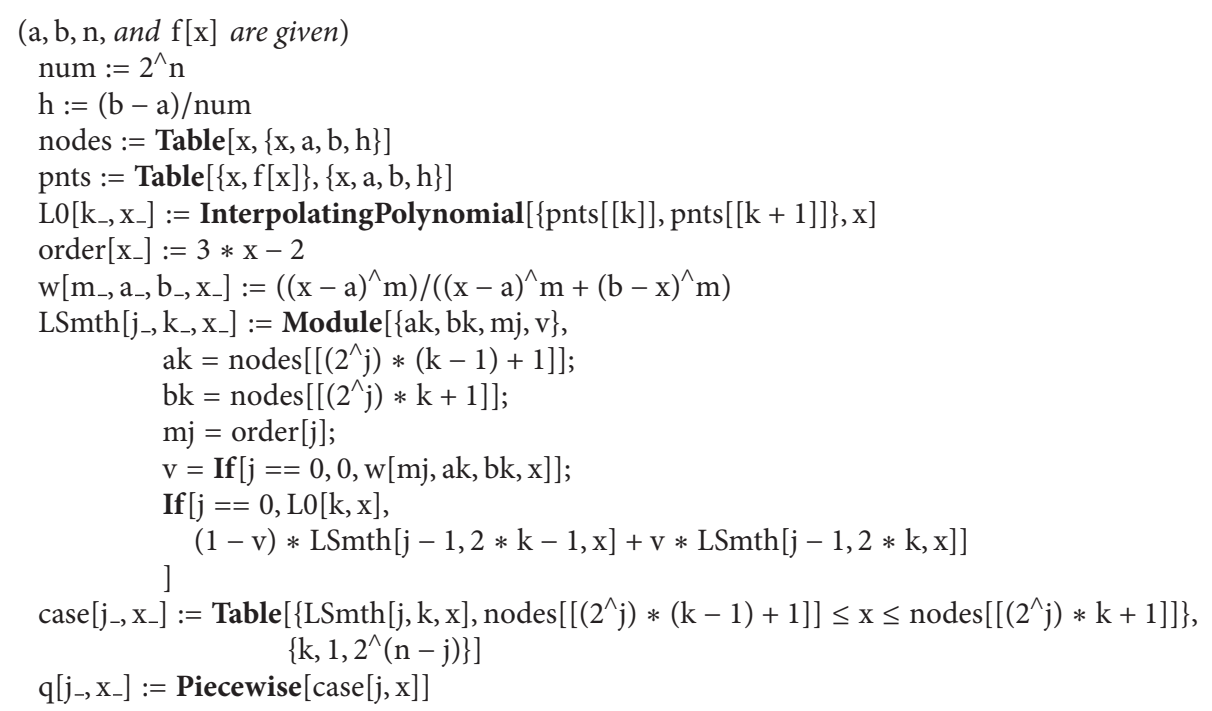

Algorithm 1: Mathematica code for the presented method.

In addition, the idea proposed in this work may be extended to the higher order piecewise polynomial interpolation.

\section{Appendix}

See Algorithm 1.

\section{Conflict of Interests}

The author declares that there is no conflict of interests regarding the publication of this paper.

\section{Acknowledgments}

The author would like to show sincere gratitude to reviewers for their helpful comments and valuable suggestions on the first draft of this paper. This research was supported by Basic Science Research program through the National Research Foundation of Korea (NRF) funded by the Ministry of Education, Science and Technology (NRF2013R1A1A4A03005079).

\section{References}

[1] L. N. Trefethen, Approximation Theory and Approximation Practice, SIAM, Philadelphia, Pa, USA, 2013.

[2] K. E. Atkinson, An Introduction to Numerial Anaysis, John Wiley \& Sons, New York, NY, USA, 2nd edition, 1989.

[3] D. Kincaid and W. Cheney, Numerial Anaysis, Brooks/Cole, Singapore, 2002.

[4] S. Prössdorf and A. Rathsfeld, "On an integral equation of the first kind arising from a cruciform crack problem," in Integral Equations and Inverse Problems, V. Petkov and R. Lazarov, Eds., pp. 210-219, Longman, Coventry, UK, 1991.
[5] D. Elliott, "Sigmoidal transformations and the trapezoidal rule," The Journal of the Australian Mathematical Society Series B: Applied Mathematics, vol. 40, pp. E77-E137, 1998/99.

[6] D. Elliott and E. Venturino, "Sigmoidal transformations and the Euler-Maclaurin expansion for evaluating certain Hadamard finite-part integrals," Numerische Mathematik, vol. 77, no. 4, pp. 453-465, 1997.

[7] B. I. Yun, "An extended sigmoidal transformation technique for evaluating weakly singular integrals without splitting the integration interval," SIAM Journal on Scientific Computing, vol. 25, no. 1, pp. 284-301, 2003. 


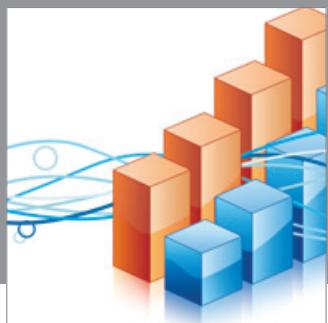

Advances in

Operations Research

mansans

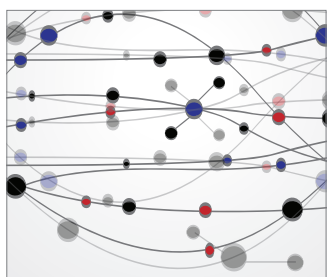

The Scientific World Journal
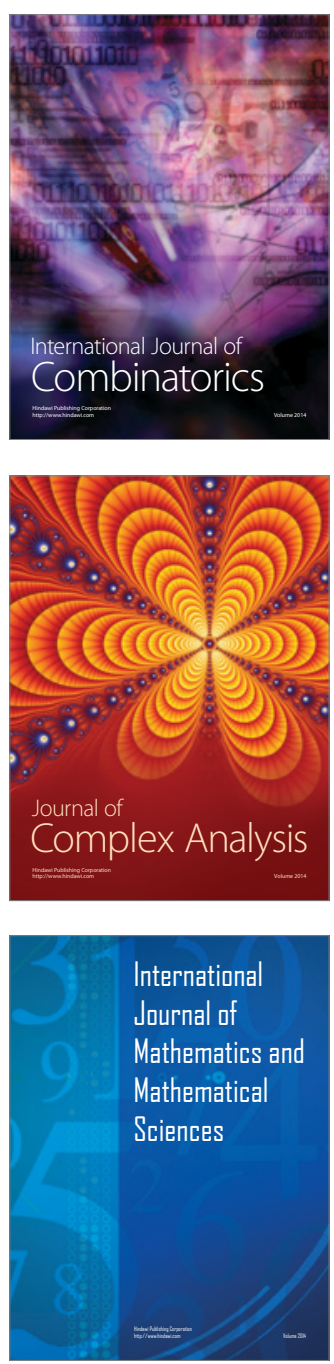
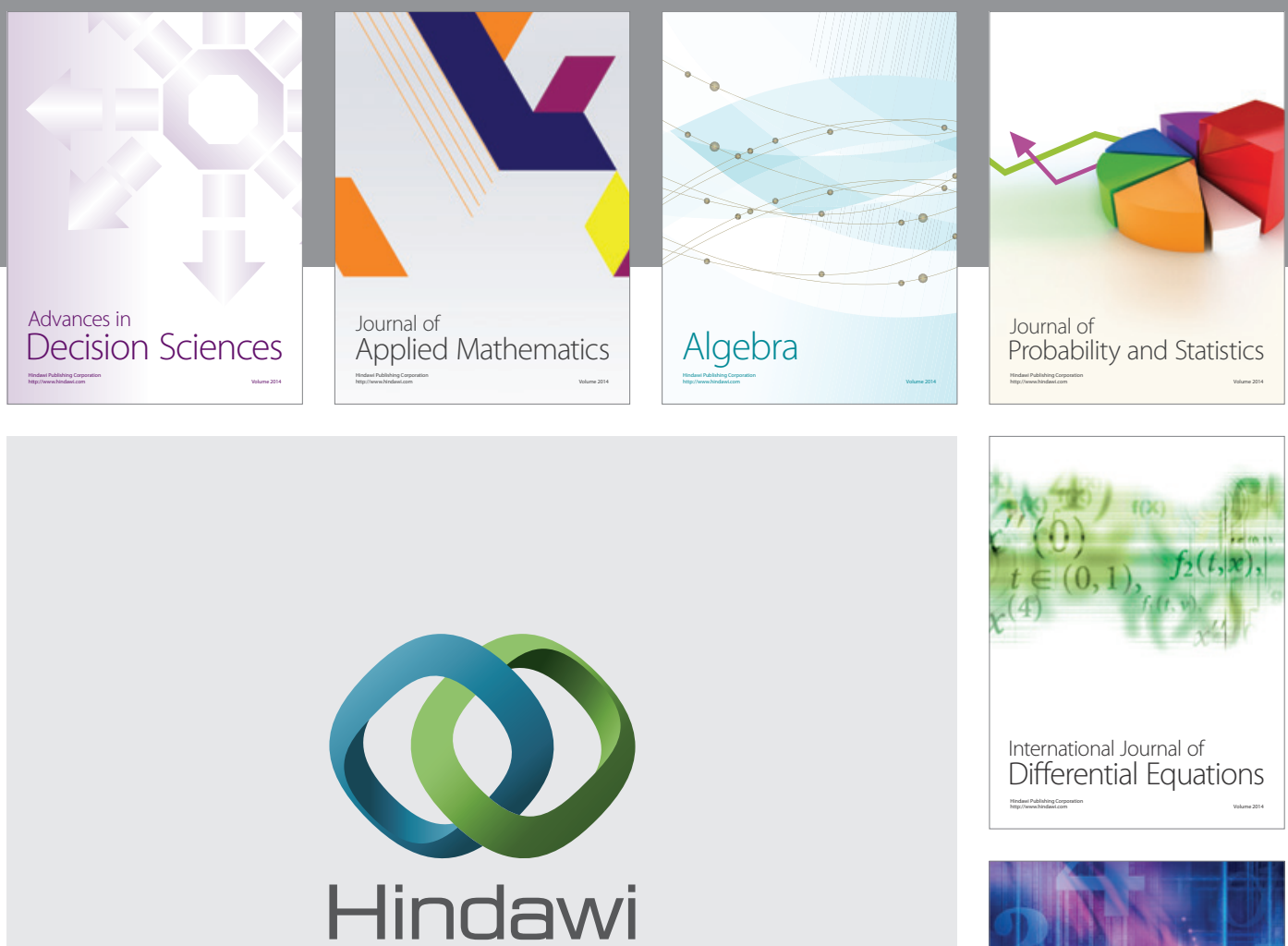

Submit your manuscripts at http://www.hindawi.com
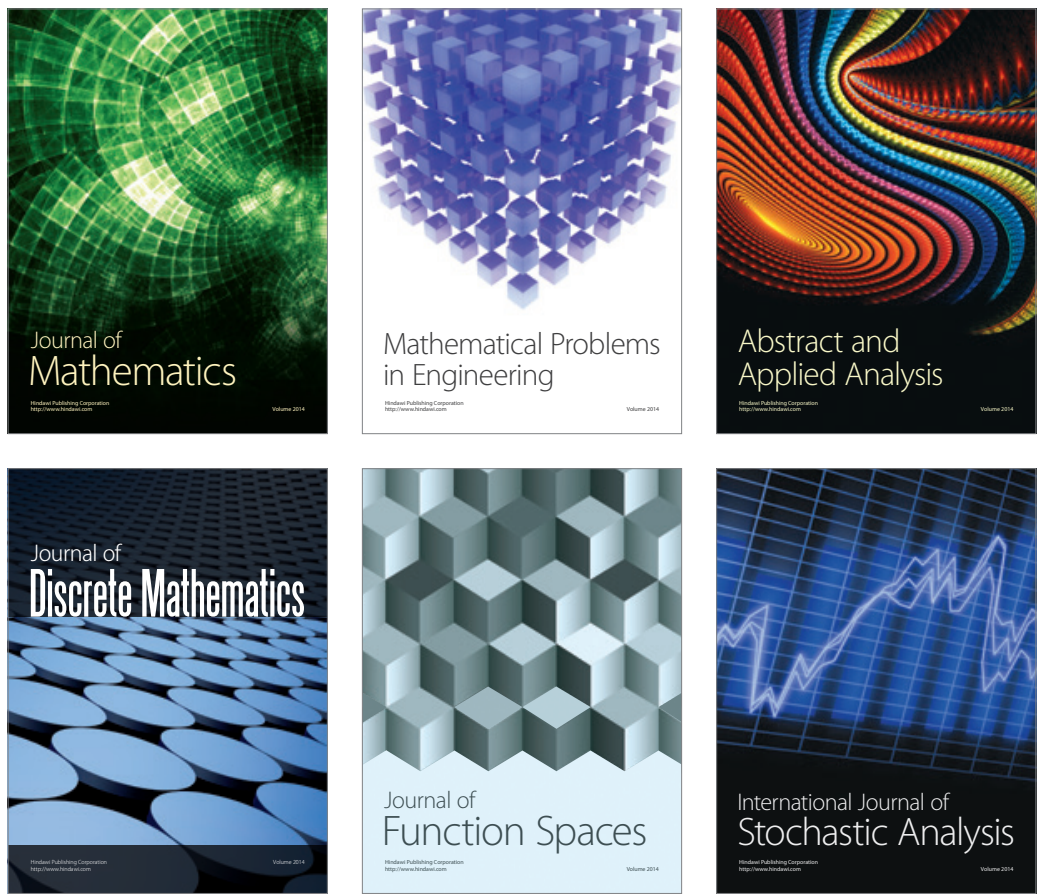

Journal of

Function Spaces

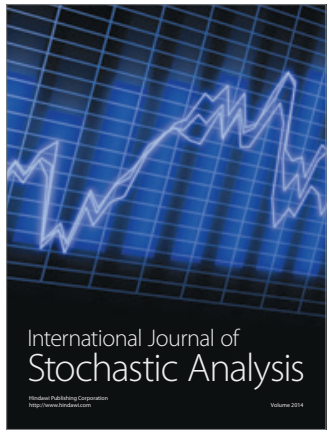

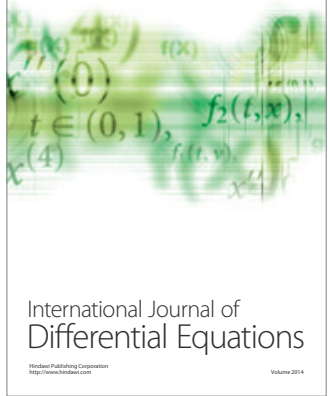
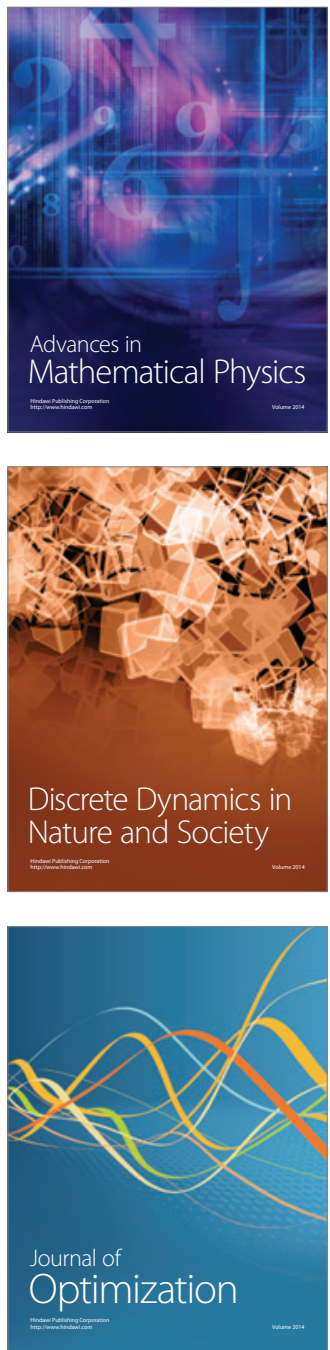